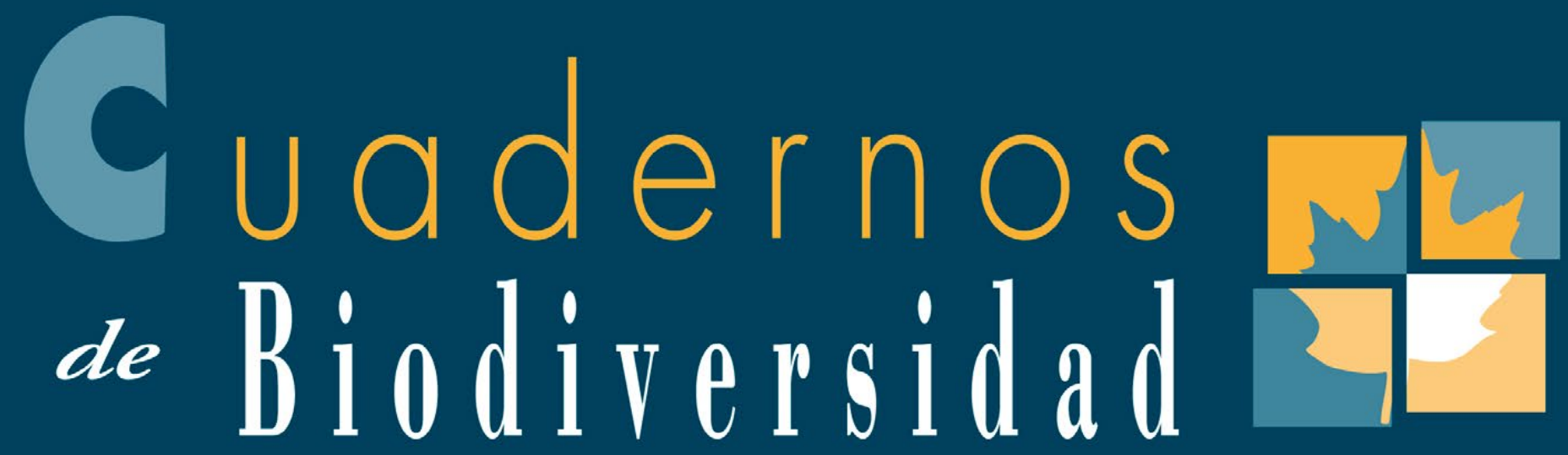

Cuadernos de biodiversidad número $49 \cdot 2015$
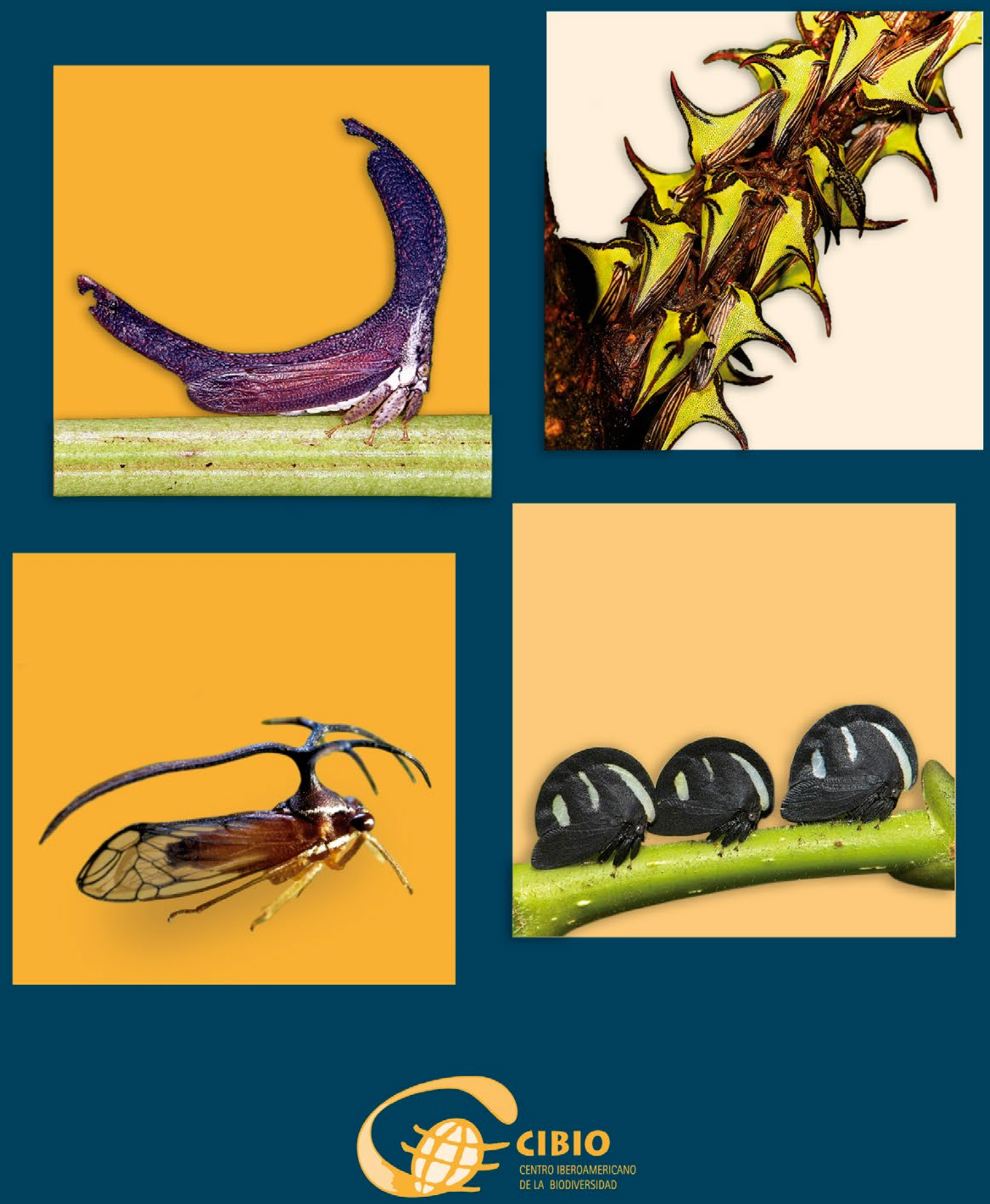

Universitat d'Alacant
Universidad de Alicante 


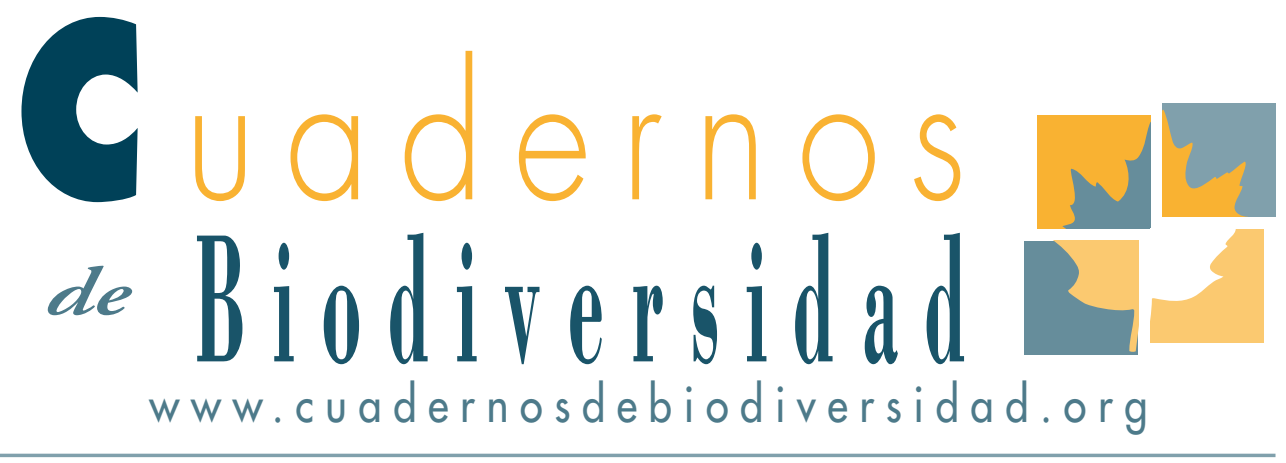

\title{
Rios saludables de Osa: una novedosa red de seguimiento de cuerpos de agua por parte de la comunidad.
}

\section{Educación ambiental participativa y acción social por la defensa de la calidad de las aguasen las comunidades de la Península de Osa, Costa Rica.}

\author{
D. Parreño Duque 1 , P. BernalCastro ${ }^{2}$ y J. Palmer ${ }^{3}$ \\ ${ }^{1}$ Investigación y Educación ambiental. Conservación Osa, Costa Rica; \\ PARRAI404@HOTMAIL.COM \\ 2 Educación Ambiental y Extensión Comunitaria. Conservación Osa, Costa Rica; \\ PILARBERNAL@OSACONSERVATION.ORG. \\ 3 Director de Ciencia y Educación. Conservación Osa, Costa Rica. \\ JIMPALMER@OSACONSERVATION.ORG.
}

\section{ABSTRACT}

The gradual deterioration of the quality of water bodies in Costa Rica has created the need to conduct periodic studies of rivers, lakes and wetlands water quality present in the Osa Peninsula, the nation's natural jewel. We work to obtain baseline data on water quality in the ACOSA region (Osa Conservation Area) involving different communities in protecting the riparian ecosystems of the watersheds in the region. All data are incorporated into a public online Water Atlas where citizens can use it as a tool to demand improvements in public systems of water management as well as to alert public health services if necessary.
Key words: Citizen Science, Water Quality, Public Health, Pollution, Workshops, Land use.

\section{INTRODUCCIÓN}

Con una superficie terrestre de apenas 51.100 $\mathrm{km} 2$, menos del 0,035\% de la superficie terrestre mundial, Costa Rica es uno de los países con mayor biodiversidad del planeta. Con cerca de 95.000 especies, registradas, alberga alrededor del 4,75\% de la biodiversidad mundial (SINAC e INBio 2009) y es considerado uno de los 20 países con mayor biodiversidad del mundo (Obando 2007). 
Sin embargo, Costa Rica presenta distintos contaminantes en sus cuerpos de agua, como basura, plaguicidas, carburantes y residuos sólidos. Estos pueden infiltrarse en el suelo contaminando el agua subterránea, así como transportarse por la escorrentía hacia las aguas superficiales (Fournier et al. 2010).

Además, las perturbaciones antropogénicas como incendios, deforestación y urbanismo, así como los malos usos del suelo provocan un exceso de sedimentos en los ríos, que conllevan a la degradación de zonas costeras como manglares, desembocaduras y arrecifes (Fournier et al. 2010).

En lo que respecta a la gestión del agua en Costa Rica, se estima que únicamente el $4 \%$ de las aguas son tratadas antes de ir a parar a ríos y otros cuerpos de agua. Casi todo lo que sale de viviendas, comercios e industria llega a los afluentes, contaminándolos con materia orgánica, sustancias químicas y metales pesados (Soto 2013). La infraestructura sigue siendo la misma desde la década del 40 y la forma de tratamiento que sigue imperando es el tanque séptico y el alcantarillado (Alfaro 2012); se estima que 25 de las 34 cuencas hidrográficas de Costa Rica están contaminadas con materia fecal, sustancias químicas y basura (CGR 2013).

En la Península de Osa la contaminación de los cuerpos de agua se debe principalmente a los elevados niveles de bacterias coliformes presentes en ellos, haciendo que la utilización del agua para fines recreativos de contacto primario, la acuacultura o el riego de cultivos no se recomiende. En la mayoría de los casos, estos niveles exceden el límite máximo permitido que establecen muchos reglamentos nacionales (Mora y Calvo2012).

Por consiguiente, existe cierta urgencia a la hora de trabajar por la calidad de los distintos cuerpos de agua de la Península de Osa, así como de movilizar a las diversas comunidades que la conforman para que exijan a los políticos mejoras en los sistemas de gestión pública del agua. El proyecto Ríos Saludables de Osa (RSO) acerca al ciudadano una poderosa herramienta informativa con el fin de que la utilice para mejorar su salud pero también la de su entorno, contribuyendo a la conservación de las cuencas hidrográficas de las que forma parte.

Siguiendo el modelo "Adopt-A-Stream", que desde el año 2002 lleva a cabo estudios periódicos de cuerpos de agua en Georgia (Estados Unidos), RSO proporciona material, ayuda logística y apoyo técnico si es necesario. A través de talleres se forma a los ciudadanos, creando líderes comunales que puedan formar a su vez a otras personas.

Por todo esto, además de por el carácter público de todos los datos obtenidos, RSO es un proyecto único en Costa Rica, novedoso y potencialmente exitoso, fácilmente exportable a otras regiones del país pero también a otros países con características similares desde el punto de vista de la gestión de los recursos hídricos. Nuestro objetivo es introducir el concepto de red RSO y proporcionar un ejemplo del tipo de datos que se quieren tomar mensualmente para futuros análisis estacionales y geográficos.

\section{ÁREA DE ESTUDIO}

En la Península de Osa, subregión de la Región Pacífico Sur, el clima es de tipo lluvioso con influencia monzónica (Coen 1967, Solano 1996).

La temperatura media anual se sitúa entre los 17 a $23^{\circ} \mathrm{C}$ y la precipitación media anual es de 4282 mm con un promedio de 192 días con lluvia (Solano y Villalobos 2001). Es por ello que la vegetación presente corresponde a la de bosque húmedo no alterado, de hoja perenne y montano bajo (MEH 1953, Bolańos y Watson 1993, Solano 1996). Debido a que la geografía de la región Pacífico Sur no es muy accidentada, y por lo tanto no existen grandes pendientes, los ríos recorren grandes llanuras antes de llegar al mar.

Además, el territorio del Área de Conservación Osa (ACOSA) es extremadamente rico en especies terrestres y marinas, con numerosas especies endémicas y en peligro de extinción. La Península de Osa es considerada un Centro de Diversidad a nivel mundial y por su posición geográfica constituye un 
puente natural entre especies del norte y del sur del continente americano. En este contexto ACOSA presenta un mosaico de ecosistemas, funcionando como un importante banco genético de especies de flora y fauna (Oduber 2008).

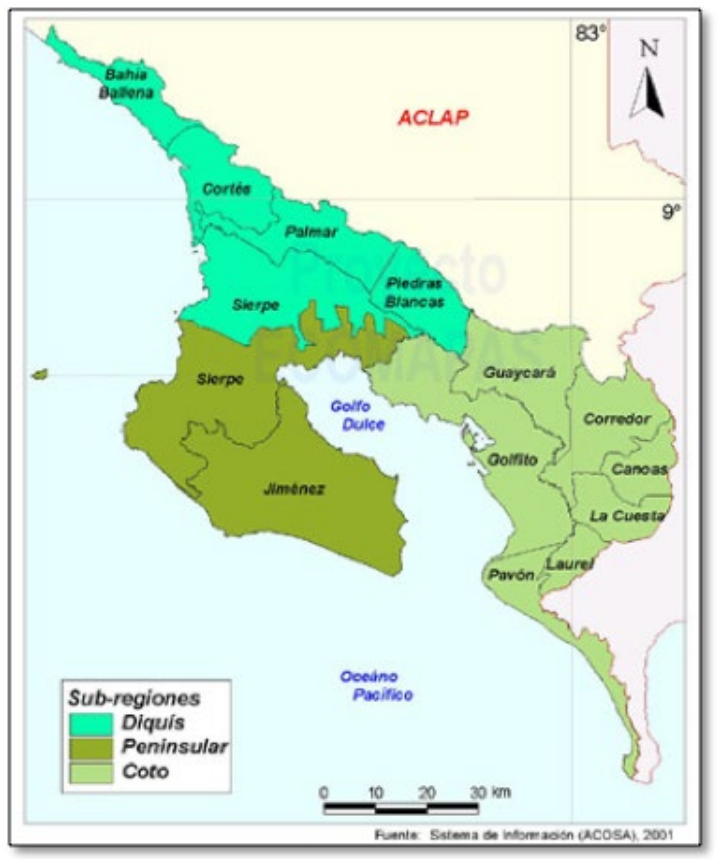

Figura 1: Territorio de ACOSA son sus subregiones administrativas.

Los muestreos fueron llevados a cabo en los siguientes lugares de ACOSA y con los siguientes grupos comunitarios:

\begin{tabular}{|l|l|l|}
\hline Grupo & Código & Cuerpo de agua \\
\hline $\begin{array}{l}\text { ASADA La } \\
\text { Palma }\end{array}$ & RNCr & Río Rincón \\
\hline $\begin{array}{l}\text { ASADA La } \\
\text { Palma }\end{array}$ & RYTr & Río Riyito \\
\hline AD La Palma & PLMq & $\begin{array}{l}\text { Quebrada La } \\
\text { Palma }\end{array}$ \\
\hline AD Carate & PHBq & Quebrada Phoebe \\
\hline $\begin{array}{l}\text { ASADA } \\
\text { Matapalo }\end{array}$ & CRBq & $\begin{array}{l}\text { Quebrada Carbo- } \\
\text { nera }\end{array}$ \\
\hline $\begin{array}{l}\text { OSACOOP } \\
\text { R. L. }\end{array}$ & VNGq & Quebrada Vanegas \\
\hline
\end{tabular}

Tabla 1: Grupos comunitarios participantes y cuerpos de agua analizados.(ASADA: Asociación Administradora de
Acueductos; AD: Asociación de Desarrollo; OSACOOP: Cooperativa de Comercialización de los Productores de Palma Aceitera de la Península de Osa R. L.)

\section{METODOLOGIAA}

Se emplean tres métodos diagnósticos para evaluar la salud de los diversos cuerpos de agua de la Península de Osa:

A) Análisis químico

B) Análisis bacteriológico

C) Análisis biológico

A) Se escogen siete tipos distintos de tests físicoquímicos:

- Temperatura del aire y del agua en ${ }^{\circ} \mathrm{C}$ (Eutech Instruments. ECTestr11 Dual Range).

- pH (La Motte. Precisión pH 3.0-10.5).

- Conductividad en $\mu S$ (Eutech Instruments. ECTestr11 Dual Range)

- Alcalinidad en mg/L (HACH ${ }^{\bullet}$. Permachem Reagents. Water Quality Test Strips).

- Nitratos en mg/L y Nitritos en mg/L (HACH • Permachem Reagents. Water Quality Test Strips).

- Oxígeno disuelto en mg/L ( $\mathrm{HACH}^{\oplus}$. PermachemReagents).

- Turbidez en JTU (LaMotte. TurbidityColumn 0-200 JTU).

B) Se utilizan placas $3 \mathrm{M}$ Petrifilm ${ }^{\mathrm{TM}}$ para analizar la presencia de bacterias coliformes en las muestras de agua. Se añade $1 \mathrm{ml}$ de muestra de agua a la placa y se incuba a una temperatura de $35+/-1{ }^{\circ} \mathrm{C}$ durante 24 a $48 \mathrm{~h}$.

C) Los macroinvertebrados son bioindicadores de la calidad del agua; en función de las familias presentes en los cuerpos de agua se obtiene un índice BMWP'-CR (Biological Monitoring Working Party modificado para Costa Rica) de calidad del agua (Vasquez et al. 2010). Este índice corresponde a seis categorías de niveles de calidad diferentes: 
- Aguas de calidad excelente.

- Aguas de calidad buena, no contaminadas o no alteradas de manera sensible.

- Aguas de calidad regular, contaminación moderada.

- Aguas de calidad mala, contaminadas.

- Aguas de calidad mala, muy contaminadas.

- Aguas de calidad muy mala, extremadamente contaminadas.

Para ello, se colocan paquetes de hojas durante al menos 3 semanas en algún lugar del cuerpo de agua que sea relativamente seguro en función de las características del mismo. Debido a las inundaciones, es difícil que estos paquetes de hojas se mantengan en su lugar en la temporada de lluvias, por lo que se aconseja esperar a la época seca.

Los paquetes de hojas son redes que contienen hojas preferiblemente de las tres especies vegetales que predominan en el lugar y que provienen de árboles con gran follaje. Estas deben de estar lo suficientemente turgentes como para que los macroinvertebrados se alimenten de ellas durante 3 semanas, por lo que deben mantenerse erectas al tomarse por el tallo. En regiones tropicales, se recomienda colocar muchas hojas pequeñas en lugar de pocas hojas grandes en las redes (SWRC 2010).

\section{RESULTADOS}

En el mes de Octubre de 2014 se llevaron a cabo dos talleres formativos que contaron con la asistencia de más de 30 personas en la Estación Biológica Piro. Uno de los talleres se centró en formar a empleados de la Asociación Conservación Osa, mientras que el otro estaba dirigido a funcionarios públicos del Gobierno (MINAET-SINAC) y distintos grupos comunitarios, como la Asociación Administradora de Acueductos (ASADA) de La Palma, la ASADA de Matapalo, las Asociaciones de Desarrollo (AD) de La Palma y Carate, y la Cooperativa de Comercialización de los Productores de Palma Aceitera de la Península de Osa (OSACOOP R.L.).
Se realizaron análisis químicos los meses de Octubre y Noviembre de 2014 con la ASADA de La Palma, la ASADA de Matapalo, las AD de La Palma y Carate y con OSACOOP R.L. en distintos lugares del Área de Conservación Osa (ACOSA) (ver Tabla 1).

Los resultados del monitoreo químico en dichos meses son los siguientes:

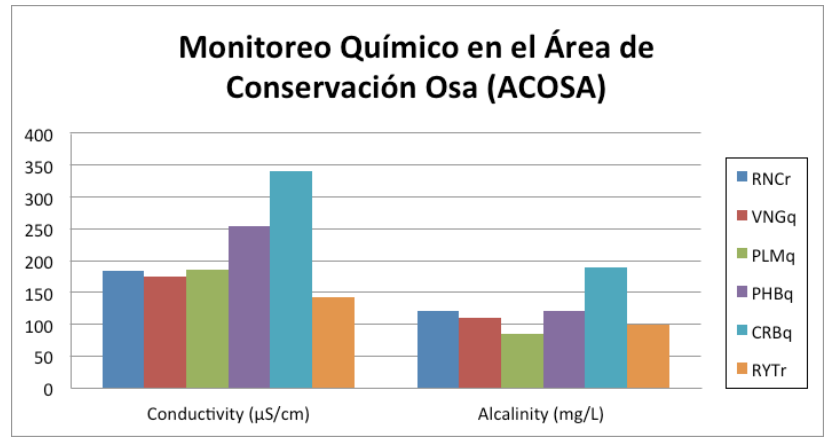

Figura 2: Conductividad $(\mu \mathrm{S} / \mathrm{cm})$ y alcalinidad $(\mathrm{mg} / \mathrm{L})$ encontradas por los grupos comunitarios en los distintos lugares del Área de Conservación Osa (ACOSA) muestreados durante los meses de Octubre y Noviembre de 2014 (RNCr:Río Rincón; VNGq:Quebrada Vanegas; PLMq: Quebrada La Palma; PHBq: Quebrada Phoebe; CRBq: Quebrada Carbonera; RYTr: Río Riyito).

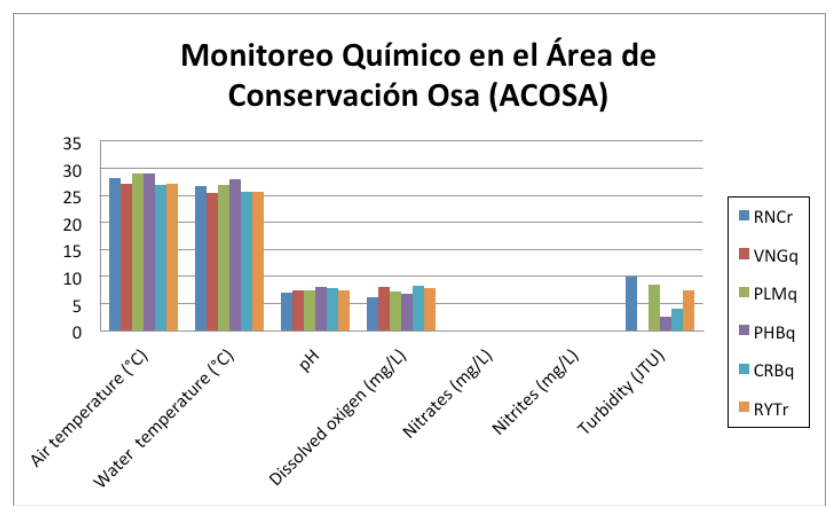

Figura 3: Temperatura del aire y del agua $\left(\mathrm{en}{ }^{\circ} \mathrm{C}\right), \mathrm{pH}$, oxígeno disuelto (en $\mathrm{mg} / \mathrm{L}$ ), nitratos (en $\mathrm{mg} / \mathrm{L}$ ), nitritos (en mg/L) y turbidez (en JTU) encontradas por los grupos comunitarios en los distintos lugares del Área de Conservación Osa (ACOSA) mustreados durante los meses de Octubre y Noviembre de 2014 (RNCr:Río Rincón; VNGq:Quebrada Vanegas; PLMq: Quebrada La Palma; PHBq: Quebrada Phoebe; CRBq: Quebrada Carbonera; RYTr: Río Riyito). 
A causa de las dificultades que entraña el trabajo de campo durante la estación de lluvias (MayoNoviembre), se decidió llevar a cabo únicamente análisis químicos de la calidad del agua. Sin embargo, la variación observada en el primer grupo de parámetros (Fig. 2) es mayor que en el segundo grupo de parámetros (Fig. 3). Se realizarán análisis bacteriológicos y macrobiológicos a partir del mes de Enero de 2015.

\section{CONCLUSIONES}

Pese a que los muestreos fueron llevados a cabo durante un corto período de tiempo y aún es pronto para sacar conclusiones relativas a la calidad del agua de los cuerpos de agua de la Península de Osa, sí se puede describir la situación actual y anticipar la situación futura en base a los resultados obtenidos

Se aprecia una mayor conductividad y alcalinidad en las quebradas Carbonera CRBq $(340 \mu \mathrm{S} /$ $\mathrm{cm})$ y Phoebe PHBq $(255 \mu \mathrm{S} / \mathrm{cm})$ que en el resto de cuerpos de agua, que se corresponde con unos niveles de $\mathrm{pH}$ mayores (7,9 y 8 respectivamente); una mayor actividad fotosintética que en el resto de ríos y quebradas, con la consiguiente liberación de grupos hidroxilo $\mathrm{HO}$ - al medio, podría explicar estos aumentos.

La turbidez es elevada en el río Rincón $\mathrm{RNCr}(10$ JTU) y en la quebrada La Palma PLMq (8,5 JTU); en el primer caso, el hecho de que se trate de un río con un caudal importante, que pasa por distintas localidades de la Península, podría explicar este valor elevado. El segundo caso es el de una quebrada de tamaño medio que sin embargo presenta una cantidad importante de partículas en suspensión; la falta de tratamiento de las aguas residuales y depuración de las mismas en la región explicaría este valor.

El carácter público de todos los datos obtenidos, así como las distintas actividades formativas organizadas con las comunidades y la flexibilidad a la hora de coordinarlas y llevarlas a cabo, hace de RSO un proyecto fácilmente exportable a otras regiones del país pero también a otros países con características similares desde el punto de vista de la gestión de los recursos hídricos.

Debido a que las distintas comunidades de la Península han respondido sorprendentemente bien a la convocatoria de Ríos Saludables de Osa y se han involucrado en el proyecto desde el primer momento, concienciándose de la necesidad de conservar las cuencas hidrográficas y luchando por la calidad de sus aguas, desde Conservación Osa esperamos servir de inspiración a otras organizaciones para que se animen a realizar labores de formación comunitaria en pos del desarrollo rural, a través de la mejora de la calidad del agua de las cuencas hidrográficas y la consiguiente conservación de las mismas.

\section{BIBLIOGRAFÍA}

Alfaro D. 2012. Decimonoveno Informe Estado de la Nación en desarrollo humano sostenible. La Política Nacional de Ordenamiento Territorial y la situación del Gran Area Metropolitana. Informe final.

Bolaños RA y Watson V. 1993. Mapa Ecológico de Costa Rica. Centro Científico Tropical. Escala 1:200.000. San José, Costa Rica.

Coen E. 1967. Algunos Aspectos sobre Climas de Costa Rica. Universidad de Costa Rica. San José, Costa Rica.

Contraloria General de la Republica (CGR). 2013. Informe acerca de la eficacia del estado para garantizar la calidad del agua en sus diferentes usos. División de fiscalización operativa y evaluativa. Área de servicios ambientales y de energía. 15 de Febrero.

Fournier ML, Ramírez F, Ruepert C, Vargas S y Echeverría S. 2010. "Diagnóstico sobre contaminación de aguas, suelos y productos hortícolas por el uso de agroquímicos en la microcuenca de las quebradas Plantón y Pacayas en Cartago, Costa Rica”. IRET Instituto Regional de Estudios en Sustancias Tóxicas y Universidad Nacional, Heredia. Informe final.

Ministerio de Economía y Hacienda (MEH). 1953. Dirección General de Estadísticas y Censos.Atlas Estadístico de Costa Rica. San José, Costa Rica. 114p.

Mora J y Calvo G. 2010. Estado actual de contaminación con coliformes fecales de los cuerpos de agua de la Península de Osa. Tecnologia en marcha. 25:34. 
Obando V. 2002. Biodiversidad de Costa Rica. Estado del Conocimiento y Gestión. Instituto Nacional de Biodiversidad, Santo Domingo de Heredia, Costa Rica. 81 p.

Oduber R. (2008). Caracterización social, ambiental, económica y legal de la cacería de animales silvestres en el sitio Osa, Costa Rica. Programa de monitoreo ecológico para la evaluación de la efectividad de las estrategias de conservación en el Area de Conservacion Osa (ACOSA). Gestión para el desarrollo humano sostenible. Informe final.

SINAC eINBio.2013. IV Informe de pais al Convenio sobre la Biodiversidad Biológica.

Solano J. 1996. Aspectos fisiográficos básicos para las principales regiones de Costa Rica. Ministerio del Ambiente y Energía, Instituto Meteorológico Nacional. San José, Costa Rica.
Solano J, Villalobos R, Instituto Meteorológico Nacional y Gestión de Desarrollo (2001). Regiones y subregiones metereologicas de Costa Rica. Instituto Nacional de Meteorologia. Costa Rica. Recuperado el 29 de Noviembre de 2014 desde http://www.imn.ac.cr/publicaciones/ estudios/Reg_climaCR.pdf.

Soto M. 2013. Solo el 4\% de las aguas residuales generadas en Costa Rica es tratado antes de ir a los ríos. La Nación, San José (CR); miércoles 20 de noviembre.

Stroud Water Research Center (SWRC). 2010. Leaf Pack Network. Manual de recursos. SWRC.

Vásquez D, Springer M y Castro A. 2010. Bioindicadores de la calidad el agua. Cuenca del Rio Tempisque. Universidad EARTH. 